\title{
Robust Linear Output Regulation Using Extended State Observer
}

\author{
Mehran Hosseini-Pishrobat $\mathbb{D}^{1},{ }^{1}$ Jafar Keighobadi, ${ }^{1}$ \\ Atta Oveisi $(\mathbb{D}),{ }^{2}$ and Tamara Nestorović $\mathbb{D D}^{2}$ \\ ${ }^{1}$ Faculty of Mechanical Engineering, University of Tabriz, 29 Bahman, Tabriz, Iran \\ ${ }^{2}$ Institute of Computational Engineering, Ruhr-Universität Bochum, Universitätsstr. 150, D-44801 Bochum, Germany \\ Correspondence should be addressed to Atta Oveisi; atta.oveisi@rub.de
}

Received 16 February 2018; Revised 31 May 2018; Accepted 5 June 2018; Published 12 August 2018

Academic Editor: Francesco Riganti-Fulginei

Copyright (C) 2018 Mehran Hosseini-Pishrobat et al. This is an open access article distributed under the Creative Commons Attribution License, which permits unrestricted use, distribution, and reproduction in any medium, provided the original work is properly cited.

\begin{abstract}
This paper presents a disturbance rejection-based solution to the problem of robust output regulation. The mismatch between the underlying plant and its nominal mathematical model is formulated by two disturbance classes. The first class is assumed to be generated by an autonomous linear system while for the second class no specific dynamical structure is considered. Accordingly, the robustness of the closed-loop system against the first disturbance class is achieved by following the internal model principle. On the other hand, in the framework of disturbance rejection control, an extended state observer (ESO) is designed to approximate and compensate for the second class, i.e., unstructured disturbances. As a result, the proposed output regulation method can deal with a vast range of uncertainties. Finally, the stability of the closed-loop system based on the proposed compound controller is carried out via Lyapunov and center manifold analyses, and some results on the robust output regulation are drawn. A representative simulation example is also presented to show the effectiveness of the control method.
\end{abstract}

\section{Introduction}

The theory of output regulation in its essence deals with the problem of tracking/disturbance rejection of a class of signals generated by autonomous dynamic systems while guaranteeing the closed-loop stability. For linear systems, this theory is well-established, and the solution of the associated control problem is obtained by the internal model principle $[1,2]$. The principle is also instrumental in addressing the problem of robust output regulation. On this basis, robust design methods have been proposed in the literature for linear systems with uncertain parameters [3-6]. These methods consider the effect of external disturbances by an exogenous signal which belongs to the solution space of a particular differential equation. According to such a differential equation, the steady-state behavior of the underlying plant should be considered to examine the solvability of the output regulation problem. After determining the steadystate forms of the plant trajectories that satisfy the tracking/disturbance rejection requirements, the internal modelbased design can be pursued [7]. By this method, a stabilizing controller that incorporates a suitable internal model of the class of the desired signals offers guaranteed asymptotic rejection/tracking of any signal from that class.

In this paper, we depart from the conventional formulation of the output regulation problem by considering the effect of disturbances that cannot be modeled by a process signal of a differential equation. As a matter of fact, in many practical applications, such modeling assumption on the disturbances is restrictive. In many systems, the disturbances are not only caused by exogenous factors, but also from endogenous factors such as structural variations, parametric uncertainty, and unmodeled dynamics. The latter factors usually appear as state-dependent disturbances in the system's mathematical model. For example, in many structural vibration control problems, the main source of the disturbance is the unmodeled higher order modes. Even though the dominant higher order modes can be obtained via suitable system identification methods, a comprehensive dynamic model for the disturbances is not feasible [8]. As another example, in many mechanical and electromechanical systems, parametric 
uncertainty, friction, and nonlinear effects bring about statedependent disturbances that are not guaranteed to belong to the solution space of any particular dynamic system [9]. On this basis, our goal in this paper is to extend the applicability of the output regulation method to such examples. To this end, we recast the output regulation problem into the active disturbance rejection framework $[10,11]$. Active disturbance rejection control (ADRC) is a robust control method for systems with large uncertainty and disturbances [10, 12-14]. The key idea of the ADRC is to treat a robust control problem as an estimation-rejection problem. More specifically, in the first step, all sorts of discrepancies between the physical system and its nominal mathematical model including parameter variations, unknown nonlinearities, and external disturbances/noises are lumped into a total disturbance term [14]. Next, an estimator referred to as extended state observer (ESO) is applied to estimate the total disturbance and then reject it in a closed-loop framework. The active disturbance rejection control offers several promising advantages over the conventional control methods including the following: (1) ADRC is an active robustification method that in comparison to classical robust techniques (based on worst-case analysis) minimizes the conservatism in the design; (2) ADRC can handle large uncertainty/disturbances from both internal and external sources; (3) ADRC does not require involved, accurate system modeling; this feature enables a disturbanceoriented design instead of a model-oriented one [12-14]. More details about the ADRC and its various engineering applications can be found in [10,14] and the recent book [13].

In light of the above discussion, active disturbance rejection provides a suitable framework to generalize the applicability of the output regulation to the systems whose disturbances do not necessarily satisfy a differential equation. The main contribution of this paper is reported in terms of combining the merits of output regulation and disturbance rejection control techniques to achieve a better tracking performance in the presence of a general class of disturbance/uncertainty. The class of considered systems is fairly general, which includes linear systems subjected to mismatched disturbances as well as state-dependent uncertainties. Following the methodology of ADRC, we lump all disturbances/uncertainties of the underlying system into a total disturbance. The total disturbance term is decomposed into two parts. The first part can be classically modeled as the solution of a differential equation while the second part has no specific dynamical structure. The modeled component of the total disturbance is handled via standard method of the output regulation theory, which entails embedding an internal model into the closed-loop system. This approach will also guarantee output tracking of the reference signals that can be generated by the internal model. The unmodeled component of the total disturbance is handled by the method of active disturbance rejection. To this end, the system dynamics is extended by appending an integrator channel that transmits the uncertainty of the unmodeled total disturbance to its time derivative. Meanwhile, an ESO is designed that continuously monitors input-output signals of the extended system to estimate the unmodeled disturbance component. By combining this estimated value with the nominal output regulation control, the mismatch disturbance rejection is achieved. We note that since the ADRC uses an integral action to model the dynamical behavior of the disturbance, the method can be construed as an approximate output regulation technique [16]. This point of view is also explained in [17], where active disturbance rejection and output regulation are combined together for an improved robust vibration control of a MEMS gyroscope. As a novelty in the disturbance rejection aspect, a new ESO design method is proposed based on the convex programming. To this end, the ESO error dynamics is presented as a Lur'e system and its stability condition is phrased in terms of linear matrix inequalities (LMIs). Additionally, the proposed ESO applies nonlinear gain approach which enhances its immunity to the measurement noises.

We note that various methods have been reported in the literature for the general problem of disturbance rejection/attenuation of control systems; for example, LMIs-based $\mathscr{L}_{2}$ disturbance attenuation for nonlinear systems with input delays [18], operator-based disturbance attenuation/rejection [19], disturbance observer-based disturbance attenuation for stochastic systems [20], disturbance rejection based on the equivalent-input-disturbance approach [21], ESO-based disturbance rejection controller for fully actuated EulerLagrange systems [22], and sliding mode observer/controller hybrid control structure [15]. With respect to these methods, the main advantages of our method lie in combining the strong points of the output regulation and the disturbance rejection techniques. The output regulation component enables a guaranteed asymptotic rejection/tracking of the disturbances/reference signals with known dynamics. Moreover, for such disturbances/reference signals, no assumptions on the energy boundedness nor control matching are required. For disturbances without known dynamic behavior, the ESObased disturbance rejection engages to fortify the output regulation. In other words, our method attempts to get the most out of the available information about the disturbances with the primary aim of disturbance rejection rather than disturbance attenuation.

The remainder of this paper is organized as follows. In Section 2, the control problem is formulated in terms of the output regulation theory. In Section 3, a nominal regulator is designed to handle the tracking problem as well as the rejection of the disturbance components with known dynamic characteristics. In Section 4, to compensate for the disturbances without known characteristics, an ESO is developed. A convex optimization-based design method for the ESO is proposed in Section 5. The stability of the closed-loop system is investigated in Section 6. In Section 7, a simulation example based on the plotter system is elaborated. Finally, the paper is concluded by Section 8 .

\section{Formulation of the Control Problem}

Consider the following square state space model:

$$
\begin{aligned}
& \dot{x}(t)=A x(t)+B u(t)+\gamma(x(t), t), \\
& y(t)=C x(t),
\end{aligned}
$$


where $x(t) \in \mathbb{R}^{n}$ is the state, $u(t) \in \mathbb{R}^{m}$ is the control input, $y(t) \in \mathbb{R}^{m}$ denotes the measured performance output, and $\gamma(x(t), t) \in \mathbb{R}^{n} \times \mathbb{R}^{+} \longrightarrow \mathbb{R}^{n}$ is a sufficiently smooth (differentiable) nonlinear function representing the total disturbance. $A, B$, and $C$ are real matrices of appropriate dimensions satisfying the following assumption.

Assumption 1. The pair $(A, B)$ is controllable and $(C, A)$ is observable.

The control goal is to asymptotically track a given reference signal, i.e., $y_{m}(t) \in \mathbb{R}^{m}$, while boundedness of the all other signals is guaranteed in the presence of the total disturbance $\gamma(x(t), t)$. The reference signal $y_{m}(t)$ is considered to be generated by the solution of a fixed linear autonomous dynamic system of the form

$$
\begin{gathered}
\dot{w}(t)=S w(t), \\
y_{m}(t)=C_{m} w(t),
\end{gathered}
$$

where $w(t) \in \mathbb{R}^{r}$ and $S$ and $C_{m}$ are real-valued matrices with proper dimensions. The following assumption is taken to guarantee the persistence and boundedness of the reference signal $y_{m}(t)[23]$.

Assumption 2. All eigenvalues of the matrix $S$ have zero real parts with multiplicity one in the minimal polynomial.

By setting $\sigma(t):=\gamma(x(t), t)$, the system in (1) can be rewritten as

$$
\begin{aligned}
& \dot{x}(t)=A x(t)+B u(t)+\sigma(t), \\
& y(t)=C x(t) .
\end{aligned}
$$

The systems in (1) and (2) are trajectory equivalent in the sense that, under the same initial values, their state trajectories coincide with each other $[24,25]$. Therefore, we carry out the controller design and analysis on the basis of (2).

The conventional output regulation theory is based on the assumption that the perturbing disturbances belong to the solution space of the dynamic system given in (2). Here, we generalize this assumption by including disturbances which do not necessarily comply with this particular dynamics.

Assumption 3. The total disturbance signal satisfies

$$
\sigma(t)=C_{\sigma} w(t)+B \xi(t)
$$

where $C_{\sigma} \in \mathbb{R}^{r \times r}$ is a given matrix with the pair $\left(C_{\sigma}, S\right)$ being observable. The signal $\xi(t) \in \mathbb{R}^{m}$ is bounded and sufficiently differentiable such that $\dot{\xi}(t)$ is bounded as well.

Remark 4. The rationale behind Assumption 3 can be explained as follows. In many engineering systems, only the dominant frequencies of the disturbances can be obtained via time and/or frequency domain identification methods (see, for example, [8]). In such systems, one can embed the known dominant frequencies into the system (2) and model the corresponding disturbance approximation error into the term, $\xi($.$) . As another example, consider the tracking control$ problems where the dominant dynamics of the underlying systems are linear. Assuming that the norm of the statedependent disturbances is smaller than the convergence rate of the linear part, it is conceivable that, for a successful tracking, the frequency spectrum of the disturbances will contain the modes of the reference signals. Accordingly, (4) forms a suitable basis for modeling of the disturbances. An example of such modeling can be found in [9].

Let us define the tracking performance of the control system as

$$
e:=y-y_{m}
$$

Note that here and after the dependence on time variable $(t)$ is dropped (unless necessary) in the formulation for the sake of readability. The following composite system is proposed regarding the underlying output regulation control problem

$$
\begin{aligned}
& \dot{x}=A x+B u+C_{\sigma} w+B \xi, \\
& \dot{w}=S w, \\
& e=C x-C_{m} w .
\end{aligned}
$$

In order to examine the solvability of the output regulation problem, first, we consider a steady-state condition in which the output tracking is achieved. In such a steady-state condition, the trajectories of the underlying system should be welldefined to ensure that the solution of the output regulation problem exists. Denoting $\bar{x}, \bar{u}, \bar{\xi}$, and $\bar{\sigma}:=\gamma(\bar{x}, t)$, as the steady-state vectors of the state, control, unstructured, and the structured disturbances, respectively, it is easy to obtain

$$
\begin{aligned}
\dot{\bar{x}} & =A \bar{x}+B \bar{u}+\bar{\sigma}, \\
\bar{\sigma} & =C_{\sigma} w+B \bar{\xi}, \\
C \bar{x}-C_{m} w & =0 .
\end{aligned}
$$

Setting the solutions as $\bar{x}=X w$ and $\bar{u}=U w-\bar{\xi}$, for some $X \in \mathbb{R}^{n \times r}$ and $U \in \mathbb{R}^{m \times r}$, we obtain the following Sylvestertype matrix equations from (7)

$$
\begin{aligned}
X S & =A X+B U+C_{\sigma}, \\
C X-C_{m} & =0 .
\end{aligned}
$$

Theorem 5. For any given matrices $C_{m}$ and $C_{\sigma}$, there exist unique matrices $X$ and $U$ satisfying (8) if and only if the following assumption holds.

Assumption 6. For all $s \in \operatorname{spec}(S)(\operatorname{spec}($.$) represents the$ vector of eigenvalues of the matrix (.));

$$
\operatorname{rank}\left(\begin{array}{cc}
A-s I & B \\
C & 0
\end{array}\right)=n+m .
$$

Proof. See [26] for the proof. 
From a geometrical point of view, solutions of the matrix equations (8) - known as the regulator equations-corresponds to a zero-error controlled invariant manifold defined by $\mathbf{M}:=\left\{(x, w) \in \mathbb{R}^{n} \times \mathbb{R}^{r} \mid x-X w=0\right\}$ for the composite system (6). In other terms, for an initial condition of the form $x(0)=X w(0)$, under the control input $\bar{u}$, the trajectory $x$ will evolve on $\mathbf{M}$ for all $t \in \mathbb{R}^{+}$and the tracking error (5) will be identically equal to zero. From this standpoint, solving the formulated output regulation problem amounts to rendering the manifold $\mathbf{M}$ globally attractive by a suitable control function [7]. Since exact dynamic characterization of the total disturbance signal is not available, we pursue practical stabilization of the manifold $\mathbf{M}$ for the composite system (6). More specifically, trajectories of the closed-loop system all should converge to a small compact set around $\mathbf{M}$ by approximately recovering the solutions of the regulator equations. To this end, we propose a disturbance rejection-based, two-degrees-of-freedom control structure. The control system is composed of a nominal output regulator equipped with an ESO for disturbance rejection. Accordingly, the main design stages of this control system are as follows.

Stage 1. The nominal controller solves the output regulation problem for the given dynamical system in (5) with the difference that the state equation is simplified to

$$
\dot{x}=A x+B u_{n}+C_{\sigma} w .
$$

Note that the corresponding control input $u_{n} \in \mathbb{R}^{m}$ is designed to drive the nominal closed-loop trajectories toward $\mathbf{M}$ by recovering the nominal part of the steady-state control signal defined by $\bar{u}_{n}:=U w$. This corresponds to the feedforward control input required for tracking $y_{m}$ as well as compensating for $C_{\sigma} w$.

Stage 2. On the other hand, the disturbance rejection loop utilizes an ESO that continuously monitors the input-output signals of the plant to provide an estimate of the disturbance, denoted by $\widehat{\xi}$. The obtained estimated value is then integrated with the nominal control input to cancel out the disturbance signal. Accordingly, the overall control input is in the form

$$
u=u_{n}-\widehat{\xi}
$$

The configuration of the proposed control system is schematically illustrated by the block diagram in Figure 1.

\section{Nominal Output Regulation}

To solve the nominal output regulation problem, we characterize the distance of the system trajectories from the target manifold $\mathbf{M}$ by a suitable coordinate. Then, by controlling this coordinate to zero, the output regulation will be accomplished. Considering the steady-state system trajectories on the manifold, an intuitive candidate for such a coordinate is $x-\bar{x}$. However, owing to the direct dependence of the regulator equations on the solutions of $\hat{\xi}$, this method is sensitive to perturbation (see [7]). Consequently, the intuitive candidate may render the control system vulnerable to the disturbance

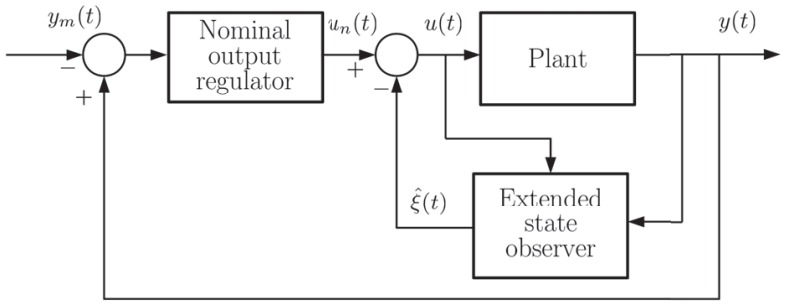

FIGURE 1: Block diagram of the proposed control system.

estimation errors. Hence, we follow a more robust design approach based on the internal model principle. For this purpose, setting $p:=d / d t$, the following linear differential operator is considered:

$$
\Gamma(p)=p^{l}+\sum_{i=0}^{l-1} \alpha_{l-i-1} p^{l-i-1}
$$

where $\left.\alpha_{i}\right|_{i=0} ^{l-1}$ with $\alpha_{l}:=1$ are the coefficients of the minimal polynomial of the matrix $S$ in the ascending order. Next, we introduce the following transformed variable:

$$
x_{t}=\Gamma(p) x
$$

Proposition 7. $x_{t} \equiv 0$, if and only if $(x, w) \in \mathbf{M}$.

Proof. The motion of the system trajectories on $\mathbf{M}$ under the control input $\bar{u}_{n}$ is equivalent to the immersion of system (10) into (2). By properties of the minimal polynomial, for any trajectory of $w$ in (2), we have $\Gamma(p) w=0$. Thereby, the set of state trajectories evolving on the manifold $\mathbf{M}$ corresponds to the kernel of the operator $\Gamma(p)$; i.e., $\operatorname{Ker}(\Gamma())=.\{x$ : $\left.\mathbb{R}^{+} \longrightarrow \mathbb{R}^{n} \mid \Gamma() x=0.\right\}$. Thus, the variable $x_{t}$ acts as an indicator function for the set $\operatorname{Ker}(\Gamma()$.$) in the sense that a state$ trajectory $x(t)$ evolves on $\mathbf{M}$ if and only if its transformation $x_{t}(t)$ is equivalent to zero.

In view of Proposition 7, the variable $x_{t}$ is a distance coordinate characterizing attractivity of the manifold $\mathbf{M}$ for trajectories of system (10). By embedding the internal model of the reference signal, this distance coordinate does not depend on the solutions of the regulator equation and, therefore, enables a robust design. Interestingly, based on this argument, the output regulation problem entails the problem of regulating $x_{t}$ to zero. Hence, by applying the operator (12) to the nominal state equation (10), we obtain the following dynamics:

$$
\dot{x}_{t}=A x_{t}+B u_{t}
$$

in which the following control transformation is introduced:

$$
u_{t}:=\Gamma(p) u_{n}
$$

Since the variable $x_{t}$ is not available for feedback, system (14) cannot be stabilized by the standard methods. To overcome this issue, we obtain the following differential equation 
describing the dynamic relation between the distance coordinate and the reference tracking through applying $\Gamma(p)$ to the tracking error (5):

$$
e^{(l)}+\sum_{i=0}^{l-1} \alpha_{l-i-1} e^{(l-i-1)}=C x_{t}
$$

This differential equation is converted to the following state space representation simply by considering $x_{t}$ as an input:

$$
\begin{aligned}
\dot{x}_{e} & =\Lambda x_{e}+E C x_{t}, \\
e & =G x_{e},
\end{aligned}
$$

in which $x_{e}:=\operatorname{col}\left(e, \ldots, e^{(l-1)}\right)$ and

$$
\begin{aligned}
\Lambda & =\left[\begin{array}{cccc}
0 & I & \ldots & 0 \\
0 & 0 & \ldots & 0 \\
\vdots & \vdots & \ddots & I \\
-\alpha_{0} I & -\alpha_{1} I & \ldots & -\alpha_{l-1} I
\end{array}\right], \\
E & =\left[\begin{array}{c}
0 \\
\vdots \\
I
\end{array}\right], \\
G & =\left[\begin{array}{llll}
I & 0 & \ldots & 0
\end{array}\right] .
\end{aligned}
$$

Setting $x_{\text {aug }}:=\operatorname{col}\left(x_{t}, x_{e}\right)$, while selecting $e$ as the fictitious output, both (14) and (17) are integrated into the following augmented system:

$$
\begin{aligned}
\dot{x}_{a u g} & =A_{a u g} x_{a u g}+B_{a u g} u_{t}, \\
e & =C_{a u g} x_{a u g},
\end{aligned}
$$

where

$$
\begin{aligned}
A_{\text {aug }} & =\left[\begin{array}{cc}
A & 0 \\
E C & \Lambda
\end{array}\right], \\
B_{\text {aug }} & =\left[\begin{array}{l}
B \\
0
\end{array}\right], \\
C_{\text {aug }} & =\left[\begin{array}{ll}
0 & G
\end{array}\right] .
\end{aligned}
$$

Proposition 8. Under Assumptions 1 and 6, the augmented system (19) is both controllable and observable.

Proof. According to the Popov-Belevitch-Hautus (PBH) test, the following rank conditions should be verified for all $s \in$ $\operatorname{spec}\left(A_{\text {aug }}\right)$; i.e.,

$$
\begin{array}{r}
\operatorname{rank}\left(\left[s I-A_{\text {aug }} B_{\text {aug }}\right]\right)=n+m l, \\
\operatorname{rank}\left(\left[\begin{array}{c}
s I-A_{\text {aug }} \\
C_{\text {aug }}
\end{array}\right]\right)=n+m l .
\end{array}
$$

Controllability. To assure the controllability of the augmented system (19), the rank condition (21) should hold. By expanding the corresponding matrix and after an appropriate rearrangement of the block rows and columns, the rank condition is inherited over

$$
\left.\operatorname{rank}\left(\left[\begin{array}{cccccc}
B & s I-A & 0 & 0 & \ldots & 0 \\
0 & -C & \alpha_{0} I & \alpha_{1} I & \ldots & \left(s+\alpha_{l-1}\right) I \\
0 & 0 & s I & -I & \ldots & 0 \\
\vdots & \vdots & \vdots & \vdots & \ddots & \vdots \\
0 & 0 & 0 & \ldots & s I & -I
\end{array}\right]\right)\right)
$$

By the controllability condition of Assumption 1, the first block row has the rank of $n$ for all $s \in \mathbb{C}$. Consequently, owing to the identity matrices with different column indices, the last $l-1$ block rows have the rank $m(l-1)$. Note that the second block row is independent of the other rows, unless for $s \in \operatorname{spec}(S)$. Thereby, to guarantee the controllability, the following rank condition needs to be satisfied for all $s \in$ $\operatorname{spec}(S)$ :

$$
\operatorname{rank}\left(\left[\begin{array}{cc}
B & s I-A \\
0 & -C
\end{array}\right]\right)=n+m
$$

Clearly, in view of Assumption 6, this condition is verified.

Observability. To show observability of the augmented system (17), the rank condition (22) needs to be verified. Again, we expand the pertinent matrix rank condition as

$$
\operatorname{rank}\left(\left[\begin{array}{ccccc}
s I-A & 0 & 0 & \ldots & 0 \\
0 & s I & -I & \ldots & 0 \\
\vdots & \vdots & \vdots & \ddots & -I \\
C & \alpha_{0} I & \alpha_{1} I & \ldots & \left(s+\alpha_{l-1}\right) I \\
0 & I & 0 & \ldots & 0
\end{array}\right]\right)
$$

Due to the observability condition of Assumption 1, the first block column has the rank $n$ for all $s \in \mathbb{C}$. The last $l$ block columns are independent due to the identity matrices with different row indices. Accordingly, the overall rank of the matrix is $n+m l$. This completes the proof.

In order to stabilize the augmented system (19), we propose a dynamic output feedback controller of the form

$$
\begin{aligned}
& \dot{x}_{\kappa}=F_{1} x_{\kappa}+F_{2} e, \\
& u_{t}=F_{3} x_{\kappa},
\end{aligned}
$$

where $x_{\kappa} \in \mathbb{R}^{n_{\kappa}}$ is the internal state of the controller and $F_{i}, i=1,2,3$ are real matrices of appropriate dimensions. The controller (26) in conjunction with the augmented system (19) yields a closed-loop system governed by the following equation:

$$
\dot{x}_{s}=A_{s} x_{s} \text {. }
$$


Here $x_{s}:=\operatorname{col}\left(x_{a u g}, x_{\kappa}\right)$ is the aggregated state vector. Moreover, the system matrix is given as

$$
A_{s}=\left[\begin{array}{cc}
A_{\text {aug }} & B_{a u g} F_{3} \\
F_{2} C_{a u g} & F_{1}
\end{array}\right] .
$$

Owing to Proposition 8, there always exist matrices $F_{1}$, $F_{2}$, and $F_{3}$ such that the resultant closed-loop system is exponentially stable. In view of this observation, without loss of generality, the following assumption is made.

Assumption 9. The controller (26) is synthesized in such a way that the matrix $A_{s}$ is Hurwitz stable.

The proposed output regulation method uses the transformed control variable (15) in its feedback loop, i.e., $u_{t}$. Consequently, the primary control input is readily available by applying the inverse of the operator $\Gamma(p)$ to the transformed variable. To this end, the following state space realization is employed:

$$
\begin{aligned}
\dot{x}_{u} & =\Lambda x_{u}+E u_{t}, \\
u & =G x_{u},
\end{aligned}
$$

where $x_{u}:=\operatorname{col}\left(u, \ldots, u^{(l-1)}\right)$ and the system matrices are the same as those of (17). The controller (26) together with the system (29) constitute the nominal output regulation loop of the overall control system.

Remark 10. System (29) replicate a model of the exogenous signal, $w$, defined by (2). Therefore, the proposed nominal output regulation has the internal model property which, in turn, guarantees asymptotic rejection/tracking of any signal belonging to the solution space of (2) [7].

\section{Extended State Observer}

The output regulation technique presented in Section 3 can handle the disturbances whose frequency spectra are embedded in the modes of system (2). For compensation of the disturbances without known frequency characteristics - represented by the signal $\xi(t)$-an ESO is designed. Note that, trivially, based on the matched condition, the unstructured disturbance is assumed to belong to the range space of $B$; otherwise the transfer function from disturbance to the output of interest cannot be set to null (see the geometrical approach proposed in [27]). Considering the composite system (6), the following ESO is proposed:

$$
\begin{aligned}
\dot{\hat{x}} & =A \widehat{x}+B u+C_{\sigma} \widehat{w}+B \widehat{\xi}+H_{1} \phi(y-\widehat{y}), \\
\dot{\widehat{w}} & =S \widehat{w}+H_{2} \phi(y-\widehat{y}), \\
\dot{\vec{\xi}} & =H_{3} \phi(y-\widehat{y}),
\end{aligned}
$$

where $\widehat{x}, \widehat{w}$, and $\widehat{\xi}$ are the estimates of the states, structured, and unstructured disturbances, respectively. Additionally, the estimated system output is $\hat{y}=C \hat{x}$. In ESO dynamics, $H_{i}, i=$ $1,2,3$, are design matrices of appropriate dimensions. Based on the nonlinear observer proposed by Prasov and Khalil [28], for positive scalars $0<\varepsilon<1$, and $d>0$, the nonlinear gain function of the observer, i.e., $\phi($.$) , is defined as$

$$
\begin{aligned}
& \phi(z)=\left[\begin{array}{ll}
\phi_{1}\left(z_{1}\right) \ldots \phi_{m}\left(z_{m}\right) & ]^{T},
\end{array}\right. \\
& \phi_{j}\left(z_{j}\right):= \begin{cases}\varepsilon z_{j}, & \left|z_{j}\right| \leq d \\
z_{j}+d(\varepsilon-1) \operatorname{sign}\left(z_{j}\right), & \left|z_{j}\right|>d, \\
j & =1, \ldots, m\end{cases}
\end{aligned}
$$

Remark 11. The gain function (31) is a dead-zone nonlinearity decreasing the observer gain when the estimation error falls inside the zone $[-d, d]$. This allows adjusting a trade-off between fast state estimation and robustness to measurement noises.

In order to investigate convergence of the ESO (30), defining the error variables as $\eta_{1}:=x-\widehat{x}, \eta_{2}:=w-\widehat{w}$, and $\eta_{3}:=\xi-\widehat{\xi}$, we obtain the following differential equation for the estimation error dynamics:

$$
\begin{aligned}
& \dot{\eta}=A_{0} \eta+H \phi(\varsigma)+Q \dot{\xi}, \\
& \varsigma=C_{0} \eta,
\end{aligned}
$$

where $\eta:=\operatorname{col}\left(\eta_{1}, \eta_{2}, \eta_{3}\right)$ and the system matrices are given by

$$
\begin{aligned}
A_{0} & =\left[\begin{array}{ccc}
A & C_{\sigma} & B \\
0 & S & 0 \\
0 & 0 & 0
\end{array}\right], \\
H & =\left[\begin{array}{c}
-H_{1} \\
-H_{2} \\
-H_{3}
\end{array}\right], \\
Q & =\left[\begin{array}{l}
0 \\
0 \\
I
\end{array}\right], \\
C_{0} & =\left[\begin{array}{lll}
C & 0 & 0
\end{array}\right] .
\end{aligned}
$$

Assumption 12. The unperturbed error dynamics, given in

$$
\begin{aligned}
& \dot{\eta}=A_{0} \eta+H \phi(\varsigma), \\
& \varsigma=C_{0} \eta,
\end{aligned}
$$

has a globally exponentially stable equilibrium at the origin. The feasibility of this assumption is addressed in Section 5.

Theorem 13. Under Assumptions 3 and 12, the estimation error of the ESO (30) is globally bounded and globally ultimately bounded in the sense that, after a finite transient time $T_{0}>0$, the following bound is valid:

$$
\|\eta\| \leq \rho_{0}+\beta \xi_{0}
$$

where $\rho_{0}>0$ is arbitrarily small while $\beta>0$, and $\xi_{0}:=$ $\sup _{t \geq 0,} \xi(t)$. 
Proof. By Assumption 12, there exist a positive definite function $V(\eta)$ and positive scalars $\beta_{0}, \beta_{1}$, and $\beta_{2}$ satisfying (see Theorem 5.17 of [29])

$$
\begin{aligned}
\left.\frac{\mathrm{d} V(\eta)}{\mathrm{d} t}\right|_{(32)} & \leq-\beta_{0} V(\eta), \\
\left\|\frac{\partial V(\eta)}{\partial \eta}\right\| & \leq \beta_{1} \sqrt{V(\eta)}, \\
\|\eta\| & \leq \beta_{2} \sqrt{V(\eta)} .
\end{aligned}
$$

Calculating the time derivative of $V(\eta)$ along the solution of (32) gives $\left.(\mathrm{d} V(\eta) / \mathrm{d} t)\right|_{(31)}=\left.(\mathrm{d} V(\eta) / \mathrm{d} t)\right|_{(32)}+$ $(\partial V(\eta) / \partial \eta) Q \dot{\xi} \leq-\beta_{0} V(\eta)+\beta_{1} \xi_{0} \sqrt{V(\eta)}$. By dividing both sides of the last inequality by $2 \sqrt{V(\eta)}$, invoking the comparison lemma and using (36), we obtain $\|\eta\| \leq \beta_{2}(\sqrt{V(\eta)}-$ $\left.\left(\beta_{1} / \beta_{0}\right) \xi_{0}\right) \exp \left(-\beta_{0} t / 2\right)+\beta_{1} \beta_{2} \xi_{0} / \beta_{0}$, which clearly shows the global boundedness of the estimation error. Moreover, considering the decaying exponential term, after the time $T_{0}=\left(2 / \beta_{0}\right) \log \left(\beta_{2}\left|\sqrt{V(\eta)}-\left(\beta_{1} / \beta_{0}\right) \xi_{0}\right| / \rho_{0}\right)$, the inequality (35) holds with $\beta:=\beta_{1} \beta_{2} / \beta_{0}$.

\section{ESO Design via LMIs}

The key to the convergence result of Theorem 13 is Assumption 12 , which requires the existence of a positive definite function $V(\eta)$ satisfying (36). In this regard, we propose a convex programming-based method to construct the function $V(\eta)$ and also to design the gain matrices $H_{i}, i=1,2,3$ in (29). Instrumental for the development of our method, the unperturbed error dynamics (34) is considered as a Lur'e system in terms of the gain function $\phi(\varsigma)$ (which is assumed to be a sector nonlinearity).

Remark 14. The gain function $\phi($.$) belongs to the sector [1, \varepsilon]$. That is, for all $\varsigma \in \mathbb{R}^{m}$,

$$
\varepsilon \varsigma^{T} \varsigma \leq \varsigma^{T} \phi(\varsigma) \leq \varsigma^{T} \varsigma .
$$

Using (34), the sector condition (37) is equivalent to the following quadratic inequality (see [30]):

$$
\varepsilon \eta^{T} C_{0}^{T} C_{0} \eta+\phi^{T}(\varsigma) \phi(\varsigma)-(1+\varepsilon) \eta^{T} C_{0}^{T} \phi(\varsigma) \leq 0 .
$$

In the framework of quadratic stabilization, we search for a positive definite function of the form $V(\eta)=\eta^{T} P \eta$, which guarantees Assumption 12 by satisfying the first requirement of (36). Thereby,

$$
\eta^{T}\left(P A_{0}+A_{0}^{T} P+\beta_{0} P\right) \eta+2 \eta^{T} P H \phi(\varsigma) \leq 0
$$

We should note that since $V(\eta)$ is a quadratic function, the other two requirements of (36) are trivially satisfied according to Rayleigh's principle.
Theorem 15. For a given constant $\beta_{0}>0$, assume there exists a positive definite matrix $P$, a matrix $Y$ with appropriate dimension, and a scalar $\vartheta \geq 0$, satisfying the LMI

$$
\begin{aligned}
& {\left[\begin{array}{cc}
A_{0}+A_{0}^{T} P+\beta_{0} P-\varepsilon \vartheta C_{0}^{T} C_{0} & Y+\vartheta\left(\frac{1+\varepsilon}{2}\right) C_{0}^{T} \\
Y^{T}+\vartheta\left(\frac{1+\varepsilon}{2}\right) C_{0} & -\vartheta I
\end{array}\right]} \\
& \quad \leq 0 .
\end{aligned}
$$

Then, the function $V(\eta)$, along with the gain matrix $H=P^{-1} Y$, guarantees the exponential stability of the origin of (34).

Proof. The origin of the system (34) is exponentially stable if the inequality (39) holds for all $\eta$ and $\phi(\varsigma)$ satisfying the sector condition (38). According to the S-procedure Lemma [30], this statement is equivalent to the existence of a scalar $\vartheta \geq 0$ such that $\eta^{T}\left(P A_{0}+A_{0}^{T} P+\beta_{0} P\right) \eta+2 \eta^{T} P H \phi(\varsigma)-$ $\vartheta\left(\varepsilon \eta^{T} C_{0}^{T} C_{0} \eta+\phi^{T}(\varsigma) \phi(\varsigma)-(1+\varepsilon) \eta^{T} C_{0}^{T} \phi(\varsigma)\right) \leq 0$. Convexifying the inequality by the transformation $Y=P H$, and using the Schur complement Lemma, LMI (40) is achieved. Thus the proof is complete.

\section{Closed-Loop Stability}

The modular structure of the proposed control system enables an independent design of the output regulation and disturbance rejection loops. However, due to the interaction of the components, the overall closed-loop system stability still needs to be investigated. The stability of the disturbance rejection loop is established by the ESO convergence results of Theorem 13 and the design method presented in Theorem 15. Therefore, we consider the effect of the ESO-based disturbance rejection on the performance of the nominal regulator. The nominal closed-loop system operating in conjunction with the disturbance rejection loop is described by the equations of the form

$$
\begin{aligned}
\dot{w} & =S w, \\
\dot{x}_{c l} & =A_{c l} x_{c l}+B_{c l} w+Q_{c l}(\xi-\widehat{\xi}),
\end{aligned}
$$

where $x_{c l}:=\operatorname{col}\left(x, x_{u}, x_{\kappa}\right)$, and

$$
\begin{aligned}
A_{c l} & =\left[\begin{array}{ccc}
A & B G & 0 \\
0 & \Lambda & E F_{3} \\
F_{2} C & 0 & F_{1}
\end{array}\right], \\
B_{c l} & =\left[\begin{array}{c}
C_{\sigma} \\
0 \\
-F_{2} C_{m}
\end{array}\right], \\
Q_{c l} & =\left[\begin{array}{l}
B \\
0 \\
0
\end{array}\right] .
\end{aligned}
$$


Additionally, to examine the effect of disturbance rejection on regulating the distance coordinate $x_{t}$, we consider system (27) perturbed by the disturbance cancellation error

$$
\dot{x}_{s}=A_{s} x_{s}+Q_{s} \xi_{t},
$$

where $\xi_{t}:=\Gamma(p)(\xi-\widehat{\xi})$ is the transformed perturbation and $Q_{s}=\left[\begin{array}{ll}B_{\text {aug }}^{T} & 0\end{array}\right]^{T}$.

Assumption 16. The signal $\xi_{t}($.$) is Lebesgue measurable and$ bounded.
The next lemma is instrumental for the closed-loop stability analysis.

Lemma 17. We have $\operatorname{spec}\left(A_{s}\right)=\operatorname{spec}\left(A_{c l}\right)$.

Proof. We show that the characteristic polynomials of $A_{s}$ and $A_{c l}$ coincide. For this, it suffices to show that for all $s \in \mathbb{C}$

$$
\operatorname{det}\left(A_{s}-s I\right)=\operatorname{det}\left(A_{c l}-s I\right) .
$$

First, regarding the block components of $A_{s}$, we have

$$
\begin{aligned}
\operatorname{det}\left(A_{s}-s I\right) & =\operatorname{det}\left(\left[\begin{array}{ccc}
A-s I & 0 & B F_{3} \\
E C & \Lambda-s I & 0 \\
0 & F_{2} G & F_{1}-s I
\end{array}\right]\right)=\operatorname{det}(A-s I) \operatorname{det}\left(\left[\begin{array}{cc}
\Lambda-s I-E C(A-s I)^{-1} B F_{3} \\
F_{2} G & F_{1}-s I
\end{array}\right]\right) \\
& =\operatorname{det}(A-s I) \operatorname{det}(\Lambda-s I) \operatorname{det}\left(F_{1}-s I+F_{2} G(\Lambda-s I)^{-1} E C(A-s I)^{-1} B F_{3}\right) .
\end{aligned}
$$

Following the same procedure for $A_{c l}$, we obtain

$$
\operatorname{det}\left(A_{c l}-s I\right)=\operatorname{det}(A-s I) \operatorname{det}(\Lambda-s I) \operatorname{det}\left(F_{1}-s I+F_{2} C(A-s I)^{-1} B G(\Lambda-s I)^{-1} E F_{3}\right)
$$

Since $G(\Lambda-s I)^{-1} E=-(1 / \Gamma(s)) I$, the matrix multiplication of $G(\Lambda-s I)^{-1} E$ commutes with $C(A-s I)^{-1} B$ and, thereby, equality (44) holds for all $s \in \mathbb{C}$.

Theorem 18. Consider the system (41). Assume that Assumptions $1-16$ are satisfied, $\lambda_{0}:=-\max _{s \in \operatorname{spec}\left(A_{c l}\right)} \operatorname{Re}(s)$, and $\xi_{t}^{0}:=$ $\sup _{\tau \geq 0}\left\|\xi_{t}(\tau)\right\|$. Then,

(1) The state trajectories $x, x_{u}$, and $x_{\kappa}$ are all globally bounded.

(2) In the state space of the composite system (6), the state trajectory $x$ converges practically to the manifold $\mathbf{M}$ in the sense that

$$
\|x-\bar{x}\| \leq \mu_{0}^{\prime}+\mu_{1}^{\prime} \exp \left(-\lambda_{0} t\right)
$$

for some positive scalars $\mu_{0}^{\prime}$ and $\mu_{1}^{\prime}$.

(3) The error variable $e$ and its first $l-1$ derivatives are globally bounded and globally ultimately bounded. That is, there is a finite time $T_{0}^{\prime \prime}>0$, after which the following inequalities holds:

$$
\left\|e^{(i)}(t)\right\| \leq \rho_{0}^{\prime \prime}+\mu_{1}^{\prime \prime} \frac{\xi_{t}^{0}}{\lambda_{0}}, \quad i=0, \ldots, l-1,
$$

where $\rho_{0}^{\prime \prime}>0$ is arbitrarily small and $\lambda_{0}>0$ is a pertinent constant.
Proof. The proof is separated into three parts.

(I) We consider the closed-loop equation (41) without perturbation

$$
\begin{aligned}
\dot{w} & =S w, \\
\dot{x}_{c l} & =A_{c l} x_{c l}+B_{c l} w .
\end{aligned}
$$

In view of Lemma 17 and Assumption 9, the matrix $A_{c l}$ is Hurwitz stable. Therefore, according to the center manifold theorem [29], there exists a stable invariant manifold $\mathbf{M}^{\prime}$ attracting all trajectories of (49). The motion of the system trajectories on $\mathbf{M}^{\prime}$ is equivalent to the immersion of the system (49) into (2). Hence, there is a linear map of the form $x_{c l}=\Pi w$, in which the matrix $\Pi$ satisfies the Sylvester equation

$$
\Pi S=A_{c l} \Pi+B_{c l} .
$$

We note that existence of $\Pi$ is guaranteed since $\operatorname{spec}(S) \cap$ $\operatorname{spec}\left(A_{c l}\right)=\emptyset$. For the perturbed closed-loop dynamics (41), an attractivity index with respect to $\mathbf{M}^{\prime}$ is defined as $q:=$ $x_{c l}-\Pi w$. Then, it is straightforward to obtain

$$
\dot{q}=A_{c l} q+Q_{c l}(\xi-\widehat{\xi}) \text {. }
$$

By Theorem 13 and following the same notation, the disturbance estimation error is bounded as $\|\xi-\widehat{\xi}\| \leq \beta \xi_{0}+$ $\eta_{0} \exp \left(-\beta_{0} t / 2\right)$, for some $\eta_{0}>0$. As a result, the solution of (51) satisfies an inequality of the form

$$
\|q\| \leq \beta \xi_{0} \mu_{0}+\mu_{1} \exp \left(-\lambda_{0} t\right)+\mu_{2} \exp \left(-\frac{\beta_{0} t}{2}\right),
$$


where $\mu_{0}, \mu_{1}$, and $\mu_{2}$ are appropriate positive numbers. Inequality (52) implies practical convergence of the closedloop trajectories to the manifold $\mathbf{M}^{\prime}$. Using the triangle inequality $\left\|x_{c l}\right\| \leq\|q\|+\|\Pi w\|$, boundedness of the trajectory $x_{c l}$ is deduced.

(II) Apportioning matrix $\Pi$ as

$$
\left[\begin{array}{l}
x \\
x_{u} \\
x_{\kappa}
\end{array}\right]=\left[\begin{array}{l}
\Pi_{x} \\
\Pi_{u} \\
\Pi_{\kappa}
\end{array}\right] w,
$$

the following matrix equation is obtained from (50):

$$
\Pi_{x} S=A \Pi_{x}+B G \Pi_{\mathcal{u}}+C_{\sigma} .
$$

Comparing (54) with (8), it follows from the uniqueness result of Theorem 5 that $X=\Pi_{x}$, and $U=G \Pi_{u}$. Thereby, $\mathbf{M}$ is a submanifold of $\mathbf{M}^{\prime}$, and finally it is implied from inequality (52) that $\|x-\bar{x}\| \leq \mu_{0}^{\prime}+\mu_{1}^{\prime} \exp \left(-\lambda_{0} t\right)$, in which $\mu_{0}^{\prime}=\beta \xi_{0} \mu_{0}+\mu_{2}$, and $\mu_{1}^{\prime}=\mu_{1}$.

(III) The solution of the state equation (43) satisfies $\left\|x_{s}(t)\right\| \leq\left(\left\|x_{s}(0)\right\|-\left\|Q_{s}\right\|\left(\xi_{t}^{0} / \lambda_{0}\right)\right) \exp \left(-\lambda_{0} t\right)+\left\|Q_{s}\right\|\left(\xi_{t}^{0} / \lambda_{0}\right)$.
Given $\rho_{0}^{\prime \prime}>0$, after the transient time $T_{0}^{\prime \prime}:=$ $\left(1 / \lambda_{0}\right) \log \left(\left|\left(\left\|x_{s}(0)\right\|-\left\|Q_{s}\right\| \xi_{t}^{0} / \lambda_{0}\right) / \rho_{0}^{\prime \prime}\right|\right)$, the inequality $\left\|x_{s}(t)\right\| \leq \rho_{0}^{\prime \prime}+\mu_{1}^{\prime \prime}\left(\xi_{t}^{0} / \lambda_{0}\right)$ holds with $\mu_{1}^{\prime \prime}=\left\|Q_{s}\right\|$. This completes the proof.

\section{Simulation Example}

In order to illustrate the efficacy of the proposed control method, we explain its application to the plotter system, which is a computer printer used for vector graphics [15]. The schematic model of the plotter is shown in Figure 2, and the corresponding symbols and numerical values are given in Table 1. The basic components of the plotter are a DC motor and three disks (referred to as disks 1-3). The state variables of the system $x_{1}, x_{2}$, and $x_{3}$ are the angular displacement of disk 1 , the angular velocity of disk 1 , and the current in the motor, respectively. The control variable is $u$ in the input voltage of the motor. Through a first principle modeling, we obtain the state equation of the plotter as follows:

$$
\dot{x}=A x+B u+\gamma(x, t),
$$

where

$$
\begin{aligned}
A & =\left[\begin{array}{ccc}
0 & 1 & 0 \\
-\frac{K_{1}+\left(r_{1} / r_{2}\right)^{2} K_{2}}{J_{1}+\left(r_{1} / r_{2}\right)^{2}\left(J_{2}+(1 / 2) M r_{2}^{2}\right)} & -\frac{B_{1}+\left(r_{1} / r_{2}\right)^{2} B_{2}}{J_{1}+\left(r_{1} / r_{2}\right)^{2}\left(J_{2}+(1 / 2) M r_{2}^{2}\right)} & \frac{K_{i}}{J_{1}+\left(r_{1} / r_{2}\right)^{2}\left(J_{2}+(1 / 2) M r_{2}^{2}\right)} \\
0 & -\frac{R_{m}}{L_{m}} & -\frac{L_{m}}{L_{m}}
\end{array}\right], \\
B & =\left[\begin{array}{c}
0 \\
0 \\
\frac{1}{L_{m}}
\end{array}\right] .
\end{aligned}
$$

The total disturbance comprises two terms in such a way that $\gamma(x, t)=\gamma_{1}(t)+B \gamma_{2}(x)$. The first, which has a known frequency spectrum, affects both motor dynamics and mechanical part of the system and is given by $\gamma_{1}(t)=[0,0.01 \sin (20 t), 0.2 \sin (20 t)]^{\top}$. The second term is a matched state-dependent uncertainty of the form $\gamma_{2}(x)=$ $-0.1 \tanh \left(10 x_{2}\right)$, which represents frictional effects in the motor. Taking the disk 1 angle as the output, $y=x_{1}$, we assume that the control objective is to track a reference signal of the form, $y_{m}(t)=0.01 \sin (t)$. According to the available frequency data of the reference/disturbance signals, system (2) is characterized by the following matrices:

$$
\begin{aligned}
S & =\left[\begin{array}{cccc}
0 & 1 & 0 & 0 \\
-1 & 0 & 0 & 0 \\
0 & 0 & 0 & 1 \\
0 & 0 & -400 & 0
\end{array}\right], \\
C_{m} & =\left[\begin{array}{llll}
1 & 0 & 0 & 0
\end{array}\right] .
\end{aligned}
$$

In view of Assumption 3 and Remark 4, we consider the disturbance model (4) with

$$
C_{\sigma}=\left[\begin{array}{llll}
0 & 0 & 0 & 0 \\
1 & 0 & 1 & 0 \\
1 & 0 & 1 & 0
\end{array}\right] .
$$

The controller (26) is designed using linear quadratic regulation in such a way that all eigenvalues of the matrix $A_{s}$ are placed on the left of the line $\mathfrak{R}(s)=-3$ in the complex plane. Parameters of the nonlinear gain function (31) are selected as $\varepsilon=0.8$ and $d=0.1$. To obtain the matrices $H_{i}, i=1,2,3$, taking $\beta_{0}=0.01$, the LMI (40) is solved per CVX [31]. We obtained

$$
H_{1}=\left[\begin{array}{c}
32.5986 \\
26.6638 \\
0.5471
\end{array}\right] \text {, }
$$


TABLE 1: Symbols of the plotter system model with the numerical values [15].

\begin{tabular}{lcc}
\hline Symbol & Description & Value \\
\hline$K_{i}$ & Motor magnetic flux & $10^{-3} \mathrm{~kg} . \mathrm{m}^{2} / \mathrm{s}^{2}$ \\
$K_{v}$ & Motor back electromotive force & $0.45 \mathrm{~V} . \mathrm{s} / \mathrm{rad}$ \\
$R_{m}$ & Motor resistance & $1 \Omega$ \\
$L_{m}$ & Motor inductance & $10^{-2} \mathrm{H}$ \\
$J_{1}$ & Disk 1 moment of inertia & $0.01213 \mathrm{~kg} . \mathrm{m}^{2}$ \\
$B_{1}$ & Disk 1 viscous friction & $0.1 \mathrm{~N} . \mathrm{m} . \mathrm{s} / \mathrm{rad}$ \\
$K_{1}$ & Disk 1 elastic constant & $10^{-3} \mathrm{~N} . \mathrm{m} / \mathrm{rad}$ \\
$r_{1}$ & Disk 1 radii & $0.1 \mathrm{~m}$ \\
$J_{2}$ & Disk 2 moment of inertia \\
$B_{2}$ & Disk 2 viscous friction & $0.01213 \mathrm{~kg} . \mathrm{m}^{2}$ \\
$K_{2}$ & Disk 2 elastic constant & $0.1 \mathrm{~N} . \mathrm{m} . \mathrm{s} / \mathrm{rad}$ \\
$r_{2}$ & Disk 2 radii & $10^{-3} \mathrm{~N} . \mathrm{m} / \mathrm{rad}$ \\
$M$ & Disk 3 (the pen nib) mass & $0.1 \mathrm{~m}$ \\
\hline
\end{tabular}

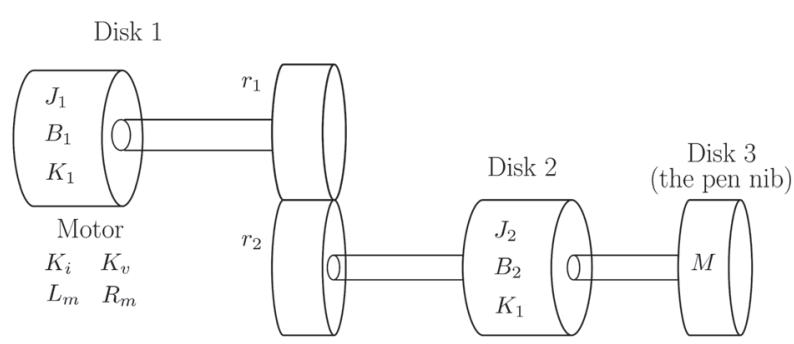

FIGURE 2: Schematics of the plotter system [15].

$$
\begin{aligned}
& H_{2}=\left[\begin{array}{c}
14.0456 \\
-4.1513 \\
-7.9373 \\
-442.8252
\end{array}\right], \\
& H_{3}=35.6611
\end{aligned}
$$

We also compare the tracking response of the controller with the hybrid controller [15], which comprises a sliding mode observer and a sliding mode output feedback controller. Since only the stabilization problem is considered in [15], we perform the following state and control transformation:

$$
\begin{aligned}
\bar{x}_{1} & =x_{1}-y_{m}, \\
\bar{x}_{2} & =x_{2}-\dot{y}_{m}, \\
\bar{x}_{3} & =x_{3}-\theta_{m}, \\
\bar{u} & =\frac{u}{L_{m}}+a_{32} \dot{y}_{m}+a_{33} \theta_{m}-\dot{\theta}_{m}, \\
\theta_{m} & =\frac{-1}{a_{23}}\left(a_{21} y_{m}+a_{22} \dot{y}_{m}-\ddot{y}_{m}\right),
\end{aligned}
$$

where $a_{i j}$ are the entries of the state matrix $A$. According to the transformation (60), the following system is obtained for the stabilization problem:

$$
\begin{aligned}
\dot{\bar{x}} & =A \bar{x}+B\left(\bar{u}+\gamma_{2}(t)\right)+\gamma_{1}(t), \\
e & =C \bar{x},
\end{aligned}
$$

where $\bar{x}=\left[\bar{x}_{1}, \bar{x}_{2}, \bar{x}_{3}\right]^{\top}$ and $C=\left[\begin{array}{lll}1 & 0 & 0\end{array}\right]$. The hybrid controller is given by

$$
\begin{aligned}
\dot{\overline{\bar{x}}}= & A \widehat{\bar{x}}+B \bar{u}+K_{h y b}(e-C \widehat{\bar{x}}) \\
& +N_{h y b} \operatorname{sign}\left(M_{h y b}(e-C \widehat{\bar{x}})\right), \\
\widehat{\gamma}_{2}= & B^{+} K_{h y b}(e-C \widehat{\bar{x}}) \\
& +B^{+} N_{h y b} \operatorname{sign}\left(M_{h y b}(e-C \widehat{\bar{x}})\right), \\
\bar{u}= & -\widehat{\gamma}_{2}-B^{+} K_{h y b} e+B^{+} N_{h y b} \operatorname{sign}\left(M_{h y b} e\right),
\end{aligned}
$$

where $\widehat{\bar{x}}$ and $\widehat{\gamma}_{2}$ are estimates of $\bar{x}$ and $\gamma_{2}$, respectively, $K_{h y b}$, $N_{h y b}$, and $M_{h y b}$ are design matrices of suitable dimensions ( $M_{h y b} C$ should be positive semidefinite), and $B^{+}$is the pseudoinverse of $B$ and is the standard signum function [15]. We note that in the design of the hybrid controller only matched disturbances are considered [15]. Using eigenvalue placement, the design matrices of the hybrid controller are tuned as follows:

$$
\begin{aligned}
K_{h y b} & =[0.0675,0.8936,1.4613]^{\top} \times 10^{3}, \\
N_{h y b} & =0.01, \\
M_{h y b} & =[1,1,1]^{\top} .
\end{aligned}
$$

The tracking responses of both controllers are shown in Figure 3. The settling times of the proposed controller and the hybrid controller are $4 s$ and $25 s$, respectively. Our controller 

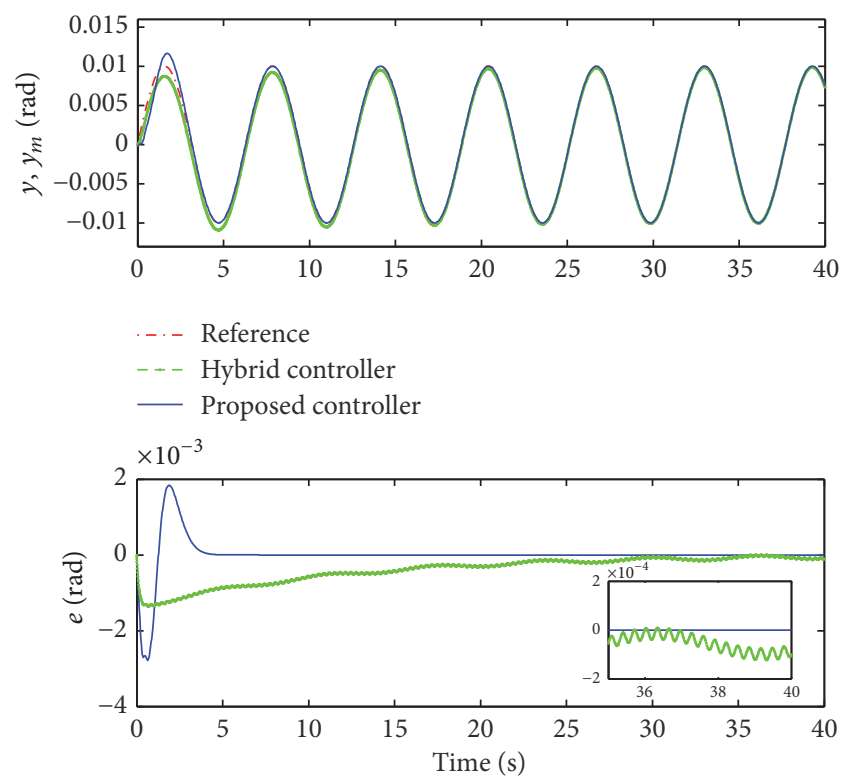

FIGURE 3: Tracking responses of the proposed controller and the hybrid controller.

shows a much faster transient response, but its overshoot is larger. The approximate values of the absolute steadystate tracking errors of the controllers are $1.567 \times 10^{-7} \mathrm{rad}$ for the proposed controller and $1.219 \times 10^{-4}$ rad for the hybrid controller. That is, our controller outperforms the hybrid controller in terms of transient time and steady-state tracking. This is mainly due to the output regulation property of our controller which enables guaranteed asymptotic tracking as well as mismatched disturbance rejection. The control signals generated by the controllers are shown in Figure 4. Both control signals are approximately the same; however, there is a high-frequency component in the control input of our controller. This component is caused by the internal model property of our controller which embeds the known frequencies of the reference/disturbances to learn their behavior [8]. In order to assess the performance of the $\mathrm{ESO}$, the estimated state variables of the plotter system are given in Figure 5. According to the ESO differential equations (30), state reconstruction is a part of disturbance estimation. The ESO successfully tracks the state variables of the system, which, in turn, confirms its disturbance estimation capability.

\section{Conclusion}

This paper presented a control structure combining output regulation and disturbance rejection control techniques. This method extends the range of the disturbances considered in the conventional output regulation theory. In the output regulation aspect, the internal model principle enables the controller to handle the mismatched disturbances with known frequency characteristics. In the disturbance rejection aspect, an ESO is employed to compensate for the disturbances without known dynamical characteristics, which, for example, may arise from nonlinear effects and

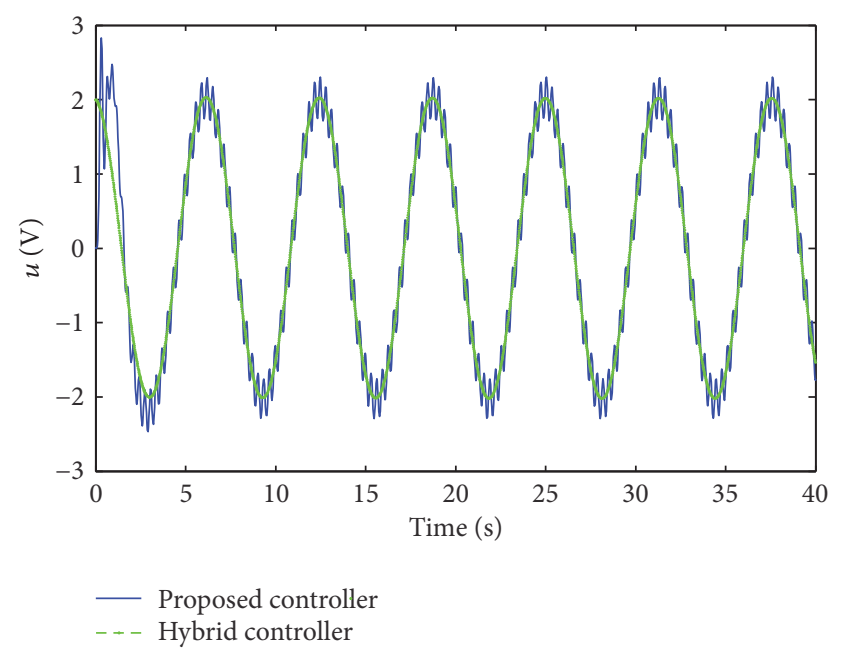

FIgURE 4: Time trajectories of the control inputs of the proposed controller and the hybrid controller.
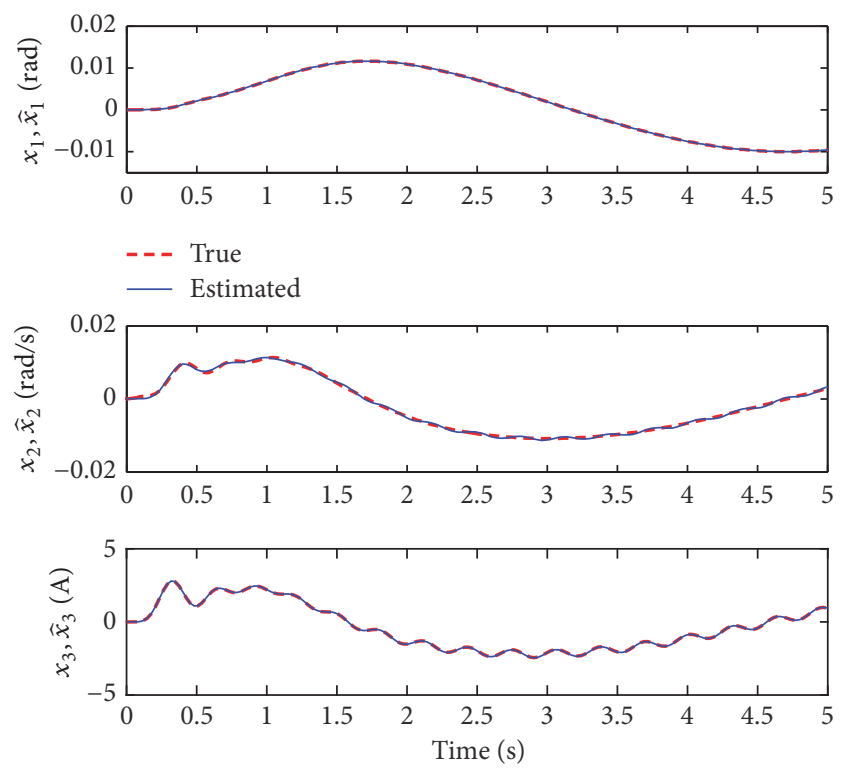

FIGURE 5: Estimation of the plotter state variables using the ESO.

structural variations of the underlying plant. Generalization and application of the method to a class of mechanical systems will be pursued in future works.

\section{Data Availability}

The authors are willing to share the implementation scripts in the form of some MATLAB m-files with the interested reader.

\section{Conflicts of Interest}

The authors declare that there are no conflicts of interest regarding the publication of this paper. 


\section{Supplementary Materials}

(1) MATLAB *.m file "Plotter_system.m" which represents the design and implementation of output regulation via the proposed NESO for a plotter system. It should be noted that the execution of this file requires the installation of CVX program which is a free disciplined convex programming tool. (2) MATLAB *.m file "Plotter_system_Hybrid_Controller.m" which represents the design and implementation of the hybrid control system on the plotter benchmark problem. (3) SIMULINK *.mdl files "Hybrid_control_system.mdl" and "Plotter_system_control.mdl" which are the realizations of closed-loop systems as SIMULINK models. (Supplementary Materials)

\section{References}

[1] B. A. Francis and W. M. Wonham, "The internal model principle of control theory," Automatica, vol. 12, no. 5, pp. 457-465, 1976.

[2] B. Francis, O. A. Sebakhy, and W. M. Wonham, "Synthesis of multivariable regulators: the internal model principle," Applied Mathematics and Optimization. An International Journal with Applications to Stochastics, vol. 1, no. 1, pp. 64-86, 1974/75.

[3] A. A. Adegbege and W. P. Heath, "Internal model control design for input-constrained multivariable processes," AIChE Journal, vol. 57, no. 12, pp. 3459-3472, 2011.

[4] E. J. Davison and A. Goldenberg, "Robust control of a general servomechanism problem: the servo compensator," Automatica, vol. 11, no. 5, pp. 461-471, 1975.

[5] J. Huang and Z. Chen, "A general framework for tackling the output regulation problem," Institute of Electrical and Electronics Engineers Transactions on Automatic Control, vol. 49, no. 12, pp. 2203-2218, 2004.

[6] A. Isidori, "Regulation and Tracking in Linear Systems," in Lectures in feedback design for multivariable systems, pp. 83-133, Springer, New York, NY, USA, 2016.

[7] Z. Chen and J. Huang, "Robust Output Regulation: A Framework," in Stabilization and Regulation of Nonlinear Systems, Z. Chen and J. Huang, Eds., pp. 197-237, Springer International Publishing, Cham, Switzerland, 2015.

[8] A. Oveisi, M. Hosseini-Pishrobat, T. Nestorović, and J. Keighobadi, "Observer-based repetitive model predictive control in active vibration suppression," Structural Control and Health Monitoring, vol. 13, no. 4, p. e2149, 2018.

[9] M. Hosseini-Pishrobat and J. Keighobadi, "Robust Vibration Control and Angular Velocity Estimation of a Single-Axis MEMS Gyroscope Using Perturbation Compensation," Journal of Intelligent Robotic Systems, vol. 32, no. 10, p. 94, 2018.

[10] Z. Gao, "On the centrality of disturbance rejection in automatic control," ISA Transactions ${ }^{\circledR}$, vol. 53, no. 4, pp. 850-857, 2014.

[11] A. Oveisi and T. Nestorović, "Robust observer-based adaptive fuzzy sliding mode controller," Mechanical Systems and Signal Processing, vol. 76-77, pp. 58-71, 2016.

[12] Z. Gao, "Active disturbance rejection control: A paradigm shift in feedback control system design," in Proceedings of the American Control Conference, 7 pages, IEEE, June 2006.

[13] B.-Z. Guo and Z.-L. Zhao, Active Disturbance Rejection Control for Nonlinear Systems, John Wiley \& Sons, Singapore, 2016.

[14] Y. Huang and W. Xue, "Active disturbance rejection control: Methodology and theoretical analysis," ISA Transactions ${ }^{\circledR}$, vol. 53, no. 4, pp. 963-976, 2014.
[15] J. D. J. Rubio, "Hybrid controller with observer for the estimation and rejection of disturbances," ISA Transactions ${ }^{\circledR}$, vol. 65, pp. 445-455, 2016.

[16] Z. Chen and D. Xu, "Output regulation and active disturbance rejection control: unified formulation and comparison," Asian Journal of Control, vol. 18, no. 5, pp. 1668-1678, 2016.

[17] M. Hosseini-Pishrobat and J. Keighobadi, "Robust output regulation of a triaxial MEMS gyroscope via nonlinear active disturbance rejection," International Journal of Robust and Nonlinear Control, vol. 28, no. 5, pp. 1830-1851, 2018.

[18] Z. Zuo, C. Wang, W. Yang et al., "Robust disturbance attenuation for a class of uncertain Lipschitz nonlinear systems with input delay," International Journal of Control, vol. 6, no. 2, pp. 1-7, 2017.

[19] M. Li and M. Deng, "Operator-based external disturbance rejection of perturbed nonlinear systems by using robust right coprime factorization," Transactions of the Institute of Measurement and Control, vol. 57, 2018.

[20] X.-J. Wei, Z.-J. Wu, and H. R. Karimi, "Disturbance observerbased disturbance attenuation control for a class of stochastic systems," Automatica, vol. 63, pp. 21-25, 2016.

[21] F. Gao, M. Wu, J. She, and W. Cao, "Disturbance rejection in nonlinear systems based on equivalent-input-disturbance approach," Applied Mathematics and Computation, vol. 282, pp. 244-253, 2016.

[22] Y. Zhang, L. Wang, J. Zhang, and J. Su, "Robust observer based disturbance rejection control for Euler-Lagrange systems," Mathematical Problems in Engineering, Art. ID 3839505 , 13 pages, 2016.

[23] A. Isidori, L. Marconi, and A. Serrani, "Fundamentals of Internal-Model-Based Control Theory," in Robust Autonomous Guidance: An Internal Model Approach, A. Isidori, L. Marconi, and A. Serrani, Eds., pp. 1-85, Springer, London, UK, 2003.

[24] C. Coleman, "Local trajectory equivalence of differential systems," Proceedings of the American Mathematical Society, vol. 16, pp. 890-892, 1965.

[25] H. Sira-Ramírez, A. Luviano-Juárez, M. Ramírez-Neria et al., "Generalities of ADRC," in Active Disturbance Rejection Control of Dynamic Systems, pp. 13-50, Elsevier, 2017.

[26] J. Huang, "Linear Output Regulation," in Nonlinear output regulation: theory and applications, pp. 1-34, SIAM, Philadelphia, $\mathrm{Pa}$, USA, 2004.

[27] A. Oveisi and T. Nestorović, "Reliability of disturbance rejection control based on the geometrical disturbance decoupling," PAMM, vol. 16, no. 1, pp. 823-824, 2016.

[28] A. A. Prasov and H. K. Khalil, "A nonlinear high-gain observer for systems with measurement noise in a feedback control framework," Institute of Electrical and Electronics Engineers Transactions on Automatic Control, vol. 58, no. 3, pp. 569-580, 2013.

[29] S. Sastry, Nonlinear Systems: Analysis, Stability, and Control, Springer, New York, NY, USA, 1999.

[30] S. Boyd, L. El Ghaoui, E. Feron et al., Linear matrix inequalities in system and control theory, society for industrial and applied mathematics, SIAM, Philadelphia, Pa, USA, 1994.

[31] M. C. Grant and S. P. Boyd, "Graph implementations for nonsmooth convex programs," in Recent Advances in Learning and Control, V. Blondel, S. Boyd, and H. Kimura, Eds., vol. 371 of Lecture Notes in Control and Information Sciences, pp. 95-110, Springer, Berlin, Germany, 2008. 


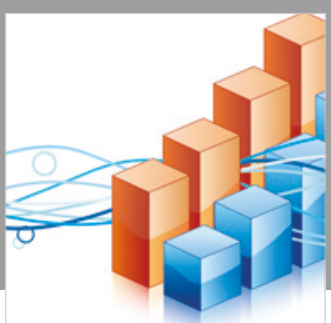

Advances in

Operations Research

\section{-n-m}
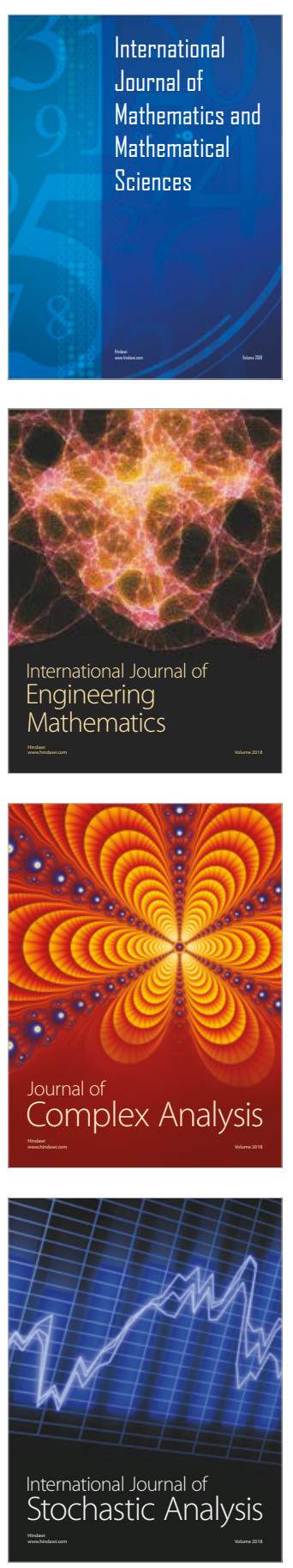
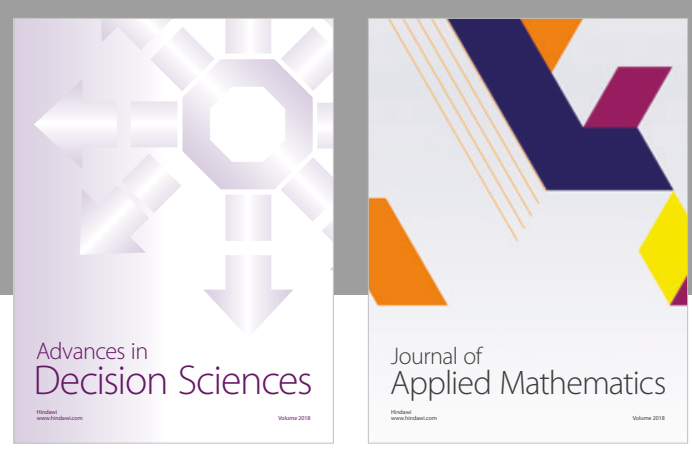

Journal of

Applied Mathematics
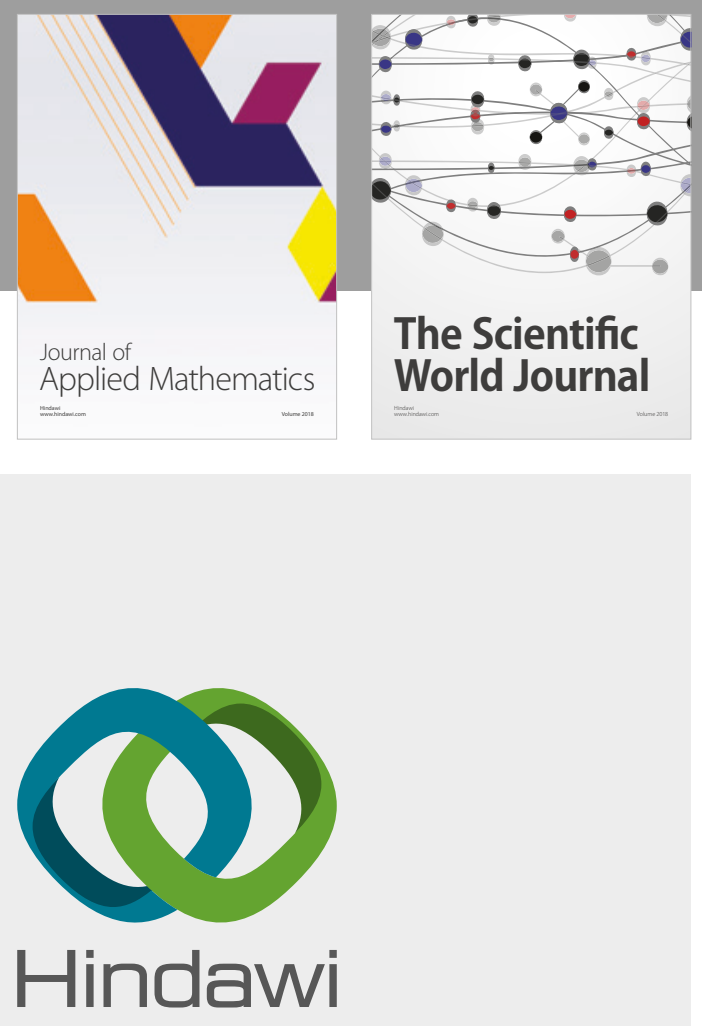

Submit your manuscripts at

www.hindawi.com

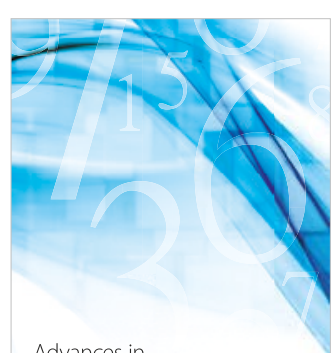

Advances in
Numerical Analysis
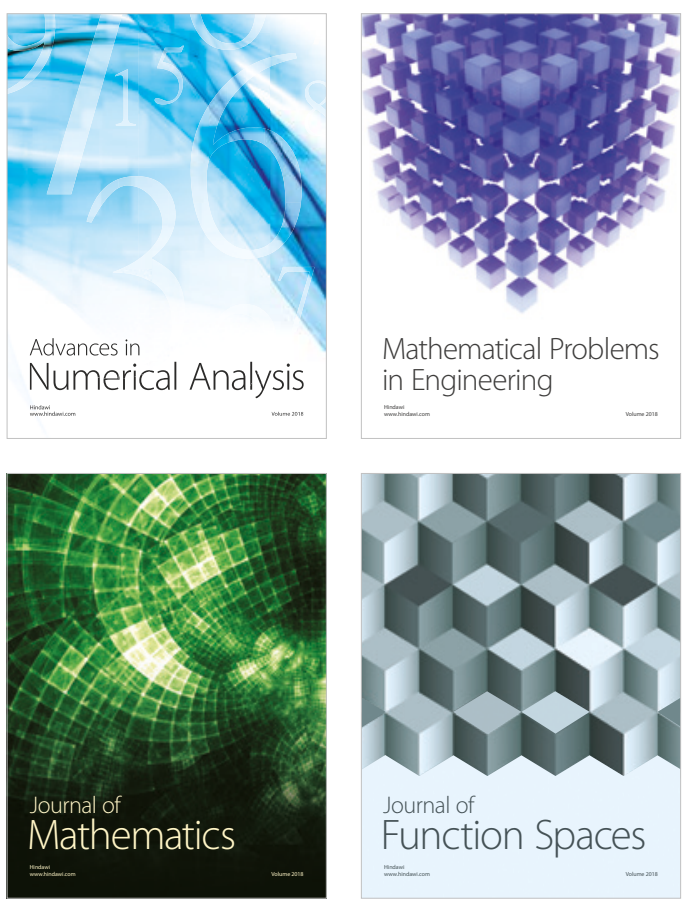

Mathematical Problems in Engineering

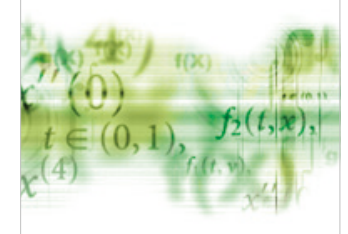

International Journal of

Differential Equations

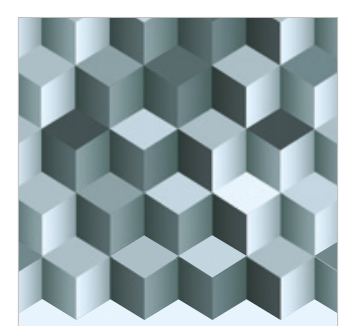

Journal of

Function Spaces

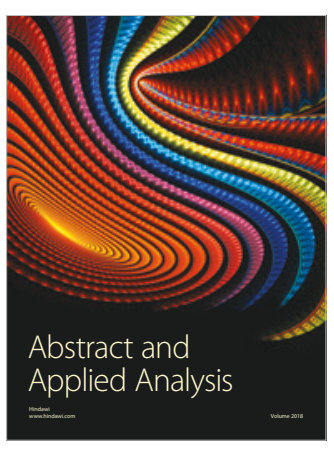

The Scientific

World Journal

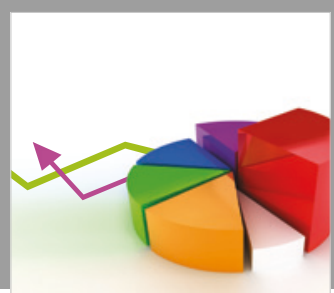

Journal of

Probability and Statistics
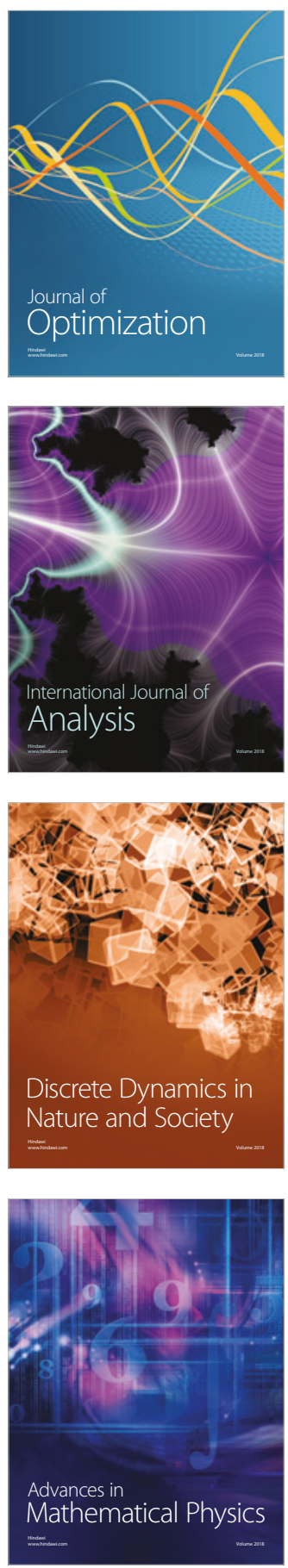